\title{
SINGULAR ELLIPTIC OPERATORS OF SECOND ORDER WITH PURELY DISCRETE SPECTRA
}

BY

ROGER T. LEWIS ${ }^{1}$

\begin{abstract}
The Friedrichs extension of a second order singular elliptic operator is considered on a weighted $\mathcal{L}_{w}^{2}(\Omega)$ space. The region $\Omega$ is not necessarily bounded. Necessary conditions and sufficient conditions on the coefficients that will insure a discrete spectrum are given with a certain degree of sharpness achieved. The boundary conditions include the Dirichlet, Neumann, and mixed Dirichlet-Neumann boundary value problems.
\end{abstract}

1. Introduction. The terminology and notation used here is essentially that used in the books by Kato [8] and Showalter [15]. The reader is referred to those books for elaboration.

Let $\mathfrak{h}$ be a symmetric closed form bounded from below by a positive number with a domain $D(\mathfrak{h})$ that is dense in a Hilbert space $H$. (In this paper $H=\mathcal{L}_{w}^{2}(\Omega)$.) The first representation theorem [8, p. 322], which was proved by Friedrichs in this special case, guarantees the existence of a selfadjoint operator $T_{\mathfrak{h}}$ associated with $\mathfrak{h}$ with the same bound from below. Moreover, $D\left(T_{\mathfrak{h}}\right)$ is a core of $\mathfrak{h}[\mathbf{8}, \mathrm{p} .317]$.

The first representation theorem of [8] applies more generally to $m$-sectorial forms $\mathrm{t}$ associated with certain operators $T_{\mathrm{t}}$ which are not necessarily selfadjoint. However, our primary concern in this paper is with conditions that will insure that $T_{\mathrm{t}}$ has a discrete spectrum, i.e., $T_{\mathrm{t}}$ has a compact resolvent and the spectrum consists of a countable number of eigenvalues of finite multiplicity which converge to $\infty$. Therefore, we may restrict our attention to $\operatorname{Re} T_{\mathrm{t}}$ since $T_{\mathrm{t}}$ has a compact resolvent if and only if $\operatorname{Re} T_{\mathrm{t}}$ has a compact resolvent [8, p. 337].

Let $\Omega$ be an open, connected subset of $\mathbf{R}^{n}$ that is not necessarily bounded. Denote the boundary of $\Omega$ by $\Gamma$. Let $w(x)$ be a measurable weight function that is positive-valued for almost every $x \in \Omega$. The Hilbert space of complex-valued functions $f(x)$ satisfying

$$
\int_{\Omega} w(x)|f(x)|^{2} d x<\infty
$$

will be denoted by $\mathcal{L}_{w}^{2}(\Omega)$. When $w(x) \equiv 1$ we denote $\mathcal{L}_{w}^{2}(\Omega)$ by $\varrho^{2}(\Omega)$.

We shall have occasion to write $\Omega$ as the union of an increasing sequence of open sets $\left\{\Omega_{k}\right\}, \Omega_{k} \subseteq \Omega_{k+1}$ for each $k$. Let $H_{\mathfrak{h}}(\Omega)$ and $H_{\mathfrak{h}}\left(\Omega_{k}\right)$ denote the inner-product

Received by the editors March 2, 1981. Presented to the UAB International Conference on Spectral Theory of Differential Operators, March 28, 1981.

1980 Mathematics Subject Classification. Primary 35P15; Secondary 35J25, 47B25, 47F05.

1 The author was supported by NSF grant number MCS-8005811. 
spaces formed from the elements of $D(\mathfrak{h})$ and $\left\{\phi 1_{\Omega_{k}}: \phi \in D(\mathfrak{h})\right\}$, respectively, with the form inner product $(u, v)_{\mathfrak{h}} \equiv \mathfrak{h}[u, v]$.

LEMMA 1. Suppose that $\Omega$ is the union of an increasing sequence of open sets $\left\{\Omega_{k}\right\}$ for which the identity injection $i_{k}: H_{\mathfrak{b}}\left(\Omega_{k}\right) \rightarrow \mathcal{L}_{w}^{2}\left(\Omega_{k}\right)$ is compact. If there is a positive-valued function $p(x)$ on $\Omega$ and a sequence of positive numbers $\varepsilon_{k} \rightarrow 0$ as $k \rightarrow \infty$ such that

$$
w(x) p(x)^{-1}<\varepsilon_{k} \text { for almost every } x \in \Omega \sim \Omega_{k}
$$

and

$$
\int_{\Omega \sim \Omega_{k}} p(x)|u(x)|^{2} d x \leqslant \mathfrak{h}[u, u] \quad \text { for all } u \in D(\mathfrak{h})
$$

then $T_{\mathfrak{h}}$ has a-discrete spectrum.

Proof. Since $T_{\mathfrak{h}}$ is bounded below by a positive number, then the mapping $T_{\mathfrak{h}}^{-1}$ : $\varrho_{w}^{2}(\Omega) \rightarrow H_{\mathfrak{h}}(\Omega)$ is continuous. Hence, we only show that the identity injection $I$ : $H_{\mathrm{b}}(\Omega) \rightarrow \varrho_{w}^{2}(\Omega)$ is compact.

Define the injection $I_{k}: H_{\mathfrak{h}}(\Omega) \rightarrow \mathcal{L}_{w}^{2}(\Omega)$ for each $k$ by $I_{k}=\beta_{k} \circ i_{k} \circ \rho_{k}$ where $\rho_{k}$ : $H_{\mathfrak{h}}(\Omega) \rightarrow H_{\mathfrak{h}}\left(\Omega_{k}\right)$ is defined by $\rho_{k} f=f 1_{\Omega_{k}}$ for $f \in H_{\mathfrak{h}}(\Omega), i_{k}$ is given by the hypothesis, and

$$
\beta_{k} f= \begin{cases}f(x), & x \in \Omega_{k}, \\ 0, & x \notin \Omega_{k} .\end{cases}
$$

Since $i_{k}$ is compact and $\beta_{k}$ and $\rho_{k}$ are continuous, then $I_{k}$ is compact.

It will suffice to show that $I$ is the limit in operator norm of the sequence $\left\{I_{k}\right\}$. Let $\phi_{k}(x) \equiv p(x)^{-1 / 2} 1_{\Omega_{k}}$ for $x \in \Omega_{k}$ and $\phi_{k} \equiv 0$ for $x \notin \Omega_{k}$; then we can write

$$
I_{k} f=\phi_{k} \sqrt{p} f
$$

for any $f \in H_{\mathfrak{h}}(\Omega)$. Consequently, if $f \in H_{\mathfrak{h}}(\Omega)$ with $\|f\|_{\mathfrak{h}}=1$, then

$$
\begin{aligned}
\left\|\left(I_{k}-I\right) f\right\|_{\mathcal{L}_{k}^{2}(\Omega)}^{2} & =\left\|\left(\phi_{k}-p^{-1 / 2}\right) p^{1 / 2} f\right\|_{\mathcal{E}_{k}^{2}(\Omega)}^{2} \\
& =\int_{\Omega \sim \Omega_{k}}\left(w(x) p(x)^{-1}\right) p(x)|f(x)|^{2} d x \\
& \leqslant \varepsilon_{k} \int_{\Omega \sim \Omega_{k}} p(x)|f(x)|^{2} d x \leqslant \varepsilon_{k} \mathfrak{h}[f, f]=\varepsilon_{k} .
\end{aligned}
$$

Therefore, $I$ is compact since it is the limit of compact operators.

Lemma 1 is known in many different special cases. See, for example, Dunford and Schwartz [3, p. 1448], Glazman [4, pp. 89, 152], and Schechter [13, p. 188]. The author is indebted to Robert M. Kauffman for communicating to him the basic idea of this proof.

The Sobolev space $H^{m}(\Omega)$ is defined to be the completion of $C^{m}(\bar{\Omega})=\left\{\left.f\right|_{\Omega}\right.$ : $\left.f \in C_{0}^{m}\left(\mathbf{R}^{n}\right)\right\}$ with respect to the norm $\|\cdot\|_{H^{m}(\Omega)}$ induced by the inner product

$$
(f, g)_{H^{m}(\Omega)}=\sum\left\{\int_{\Omega} D^{\alpha} f \cdot \overline{D^{\alpha} g}:|\alpha| \leqslant m\right\} .
$$

The space $H_{0}^{m}(\Omega)$ is the closure of $C_{0}^{\infty}(\Omega)$ in $H^{m}(\Omega)$. 
The next two hypotheses will be assumed in much of what follows. The reader is referred to Showalter [15] or Treves [16] for an explanation of some of the terms.

$\mathcal{H} 1$. Assume that $\Omega$ is the union of an increasing sequence of bounded open sets $\left\{\Omega_{k}\right\}$ each of which lies on one side of its boundary, $\Gamma_{k}$. For some positive integer $m$, assume that

$$
\left\{\phi 1_{\Omega_{k}}: \phi \in D(\mathfrak{h})\right\} \subseteq H^{m}\left(\Omega_{k}\right)
$$

for $k=1,2, \ldots$ Finally, assume that each $\Gamma_{k}$ is a $C^{m}$-manifold, and that $w(x)$ is bounded on each $\Omega_{k}$.

The next hypothesis is a coercivity requirement.

$\mathcal{H} 2$. For each $k$ there is a $c_{k}>0$ such that

$$
|\mathfrak{h}[u, u]| \geqslant c_{k}\|u\|_{H^{m}\left(\Omega_{k}\right)}^{2}
$$

for all $u \in\left\{\phi 1_{\Omega_{k}}: \phi \in D(\mathfrak{h})\right\}$.

COROLlaRY to Lemma 1. Let $\$$ be a closable, symmetric form bounded from below by a positive number. Let $\mathfrak{s}$ have closure $\mathfrak{h}$, and assume $\mathcal{H} 1$ and $\mathcal{H} 2$. If (1) of Lemma 1 holds and

$$
\int_{\Omega \sim \Omega_{k}} p(x)|v(x)|^{2} d x \leqslant \mathfrak{s}[v, v] \text { for all } v \in D(\mathfrak{s}),
$$

then $T_{\mathfrak{h}}$ has a discrete spectrum.

Proof. First, we show that (3) implies (2) of Lemma 1. If $u \in D(\mathfrak{h})$ then there is a sequence $\left\{v_{n}\right\} \in D(\mathfrak{g})$ such that $v_{n} \rightarrow u$ in $\varrho_{w}^{2}(\Omega)$ and $s\left[v_{n}-v_{m}, v_{n}-v_{m}\right] \rightarrow 0$ for $n$, $m \rightarrow \infty$. Therefore, $\left\{v_{n}\right\}$ has a limit in $\mathcal{L}_{p}^{2}\left(\Omega \sim \Omega_{k}\right)$. By (1), $u$ is the $\mathcal{E}_{p}^{2}\left(\Omega \sim \Omega_{k}\right)$-limit of $\left\{v_{n}\right\}$. Inequality (2) now follows since $\mathfrak{S}\left[v_{n}, v_{n}\right] \rightarrow \mathfrak{h}[u, u]$ as $n \rightarrow \infty$.

The identity injection from $H_{\mathfrak{h}}\left(\Omega_{k}\right)$ to $H^{m}\left(\Omega_{k}\right)$ is continuous for each $k$ by $\mathcal{H} 2$. By $\mathcal{H} 1, H^{m}\left(\Omega_{k}\right)$ can be compactly imbedded in $\varrho_{w}^{2}\left(\Omega_{k}\right)$ - see Showalter [15, p. 49]. Hence, the identity injection $i_{k}: H_{\mathfrak{h}}\left(\Omega_{k}\right) \rightarrow \mathcal{L}_{w}^{2}\left(\Omega_{k}\right)$ is compact. By Lemma 1 , the proof is complete.

A well-known application of this corollary is the one in which the potential $q(x)$ becomes unbounded at $\infty$ on an unbounded set $\Omega \subseteq \mathbf{R}^{n}$ with sufficiently smooth boundary, which implies that the spectrum of the Schrödinger operator $-\Delta_{n}+q(x)$ has a discrete spectrum $[4,13]$. The next example presents some variations on this theme. We denote the gradient operator by $\nabla$ and the Laplacian in $\mathbf{R}^{n}$ by $\Delta_{n}$.

EXAMPLE 1. Let $\Omega=\left\{x \in \mathbf{R}^{3}: x=\left(x_{1}, x_{2}, x_{3}\right)\right.$ with $x_{i}>0$ for $\left.i=1,2,3\right\}$ and

$$
\mathfrak{g}[u, v]=\int_{\Omega}[(A(x) \nabla u(x), \nabla v(x))+q(x) u(x) \overline{v(x)}] d x, \quad u, v \in D(\mathfrak{g}),
$$

where $A(x)=\left(a_{i j}(x)\right)$ is a $3 \times 3$, symmetric, positive definite matrix. Assume that $q(x)$ is real-valued and continuous on $\Omega$ and that each $a_{i j} \in C^{1}(\Omega)$. Finally, assume that the minimum eigenvalue of $A(x)$ and $q(x)$ have a positive lower bound on $\Omega$.

(i) If $D(\mathfrak{g})=\left\{u 1_{\Omega}: u \in C_{0}^{1}\left(\mathbf{R}^{3} \sim\{0\}\right)\right\}$,

$$
w(x) q(x)^{-1} \rightarrow 0 \quad \text { a.e. as }|x| \rightarrow \infty,
$$


and

$$
w(x) q(x)^{-1} \rightarrow 0 \quad \text { a.e. as }|x| \rightarrow 0
$$

then $T_{\mathrm{h}}$ has a discrete spectrum.

(ii) If $D(\mathfrak{s})=C_{0}^{1}(\Omega)$,

$$
w(x) q(x)^{-1} \rightarrow 0 \quad \text { a.e. as } x_{i} \rightarrow 0 \text { for } i=1,2,3,
$$

and

$$
w(x) q(x)^{-1} \rightarrow 0 \quad \text { a.e. as }|x| \rightarrow \infty,
$$

then $T_{\mathfrak{h}}$ has a discrete spectrum.

Proof. The fact that $s$ is closable follows from a theorem of Kato and Schechter [8, p. 318] (and it is discussed more below). The coercivity condition of $\mathscr{H} 2$ is obviously satisfied. Condition $\mathcal{H} 1$ is satisfied, and the conclusion of (i) follows by choosing, for example,

$$
\Omega_{j}=\left\{x \in \Omega: x_{1}^{1 / j}+x_{2}^{1 / j}+x_{3}^{1 / j}>1 \text { and }|x-(j, j, j)|<j\right\}
$$

for $j=2,3, \ldots$, (where $|\cdot|$ denotes the Euclidean norm). The conclusion of (ii) follows by choosing each $\Omega_{j}$ in order that $\bar{\Omega}_{j} \subset \Omega$ in a similar manner.

The form $s$ of Example 1 is associated with the elliptic differential operator

$$
L=-\sum_{i, j=1}^{3} \frac{\partial}{\partial x_{i}} a_{i j}(x) \frac{\partial}{\partial x_{j}}+q(x) .
$$

Condition (i) is satisfied by $q(x)=|x|^{-1}+|x|$ and condition (ii) is satisfied by $q(x)=x_{1}^{-1}+x_{2}^{-1}+x_{3}^{-1}+|x|$. Applications similar to (i) and (ii) follow (with appropriate adjustments in $D(\mathfrak{s}))$ when $\Omega$ is bounded and $q(x)$ approaches $+\infty$ near the boundary of $\Omega$. Also, similar applications follow for higher order elliptic operators.

We assume throughout the remainder of this paper that the boundary of $\Omega, \Gamma$, is sufficiently smooth in order that the first formula of Green applies. We denote the trace operator $\left[15\right.$, p. 40] on the Sobolev space $H^{m}(\Omega)$ by $\gamma_{0}$ and the traces of the normal derivatives by $\gamma_{j}, j=1,2, \ldots, m-1$. Let $|\cdot|$ denote the Euclidean norm in $\mathbf{R}^{n}$ (as well as absolute value - the difference being clear from the context).

The next lemma, whose origin dates to the Hardy inequality, will be used to establish condition (3). In the case of $n=1$, the proof is established in [6] for compact support functions $\phi(x)$.

LemMA 2. Let $g \in H^{2}(\Omega)$ be real-valued and satisfy $\Delta_{n} g(x) \neq 0$ on $\Omega$; then

$$
\begin{aligned}
\int_{\Omega}\left|\Delta_{n} g(x)\right||\phi(x)|^{2} d x & \leqslant 2 \int_{\Omega}|\phi(x)||\nabla g(x)||\nabla \phi(x)| d x \\
& \leqslant 4 \int_{\Omega}\left|\Delta_{n} g(x)\right|^{-1}|\nabla g(x)|^{2}|\nabla \phi(x)|^{2} d x
\end{aligned}
$$

for all $\phi(x) \in C_{0}^{\infty}\left(\mathbf{R}^{n}\right)$ that satisfy

$$
(-1)^{\eta} \int_{\Gamma} \gamma_{1} g(s) \gamma_{0}|\phi(s)|^{2} d s \leqslant 0
$$

where $\eta=0$ if $\Delta_{n} g>0$ on $\Omega$ and $\eta=1$ if $\Delta_{n} g<0$ on $\Omega$. 
Proof. By the first formula of Green,

$\int_{\Omega} \Delta_{n} g(x)|\phi(x)|^{2} d x=\int_{\Gamma} \gamma_{1} g(s) \gamma_{0}|\phi(s)|^{2} d s-2 \int_{\Omega} \nabla g(x) \cdot(\operatorname{Re} \phi(x) \nabla \overline{\phi(x)}) d x$.

Hence,

$$
\begin{aligned}
\int_{\Omega} \mid \Delta_{n} g(x) & \left.|| \phi(x)\right|^{2} d x \leqslant 2 \int_{\Omega}|\nabla g(x)||\phi(x)||\nabla \phi(x)| d x \\
& \leqslant 2\left[\int_{\Omega}\left|\Delta_{n} g(x)\right||\phi(x)|^{2} d x\right]^{1 / 2}\left[\int_{\Omega}|\nabla g(x)|^{2}\left|\Delta_{n} g(x)\right|^{-1}|\nabla \phi(x)|^{2} d x\right]^{1 / 2}
\end{aligned}
$$

by the Cauchy-Schwarz inequality. The conclusion now follows.

The lemma obviously holds for all $\phi \in C_{0}^{\infty}(\Omega)$, which will later allow us to apply it to the case of the Dirichlet problem. However, for some sets $\Omega$ and certain choices of functions $g(x)$, a much wider range of applications is possible. Since $\gamma_{1} g=\nabla g \cdot \nu$, where $\nu$ denotes the unit outward normal at $x \in \Gamma$, then $\nabla g \cdot \nu \leqslant 0$, for each $x \in \Gamma$, will insure that inequality (5) holds for all $\phi \in C_{0}^{\infty}\left(\mathbf{R}^{n}\right)$.

EXAmple 2. Suppose that, for some $\alpha \in C^{1}\left(\mathbf{R}^{1}\right)$ and $r=|x|, g(x)=\alpha(r)$ for all $x \in \Gamma$. Assume that either $\Gamma$ does not contain the origin or that $\alpha^{\prime}(0)=0$. Then, the inequality

$$
\alpha^{\prime}(r)\left(x_{1}, \ldots, x_{n}\right) \cdot \nu \leqslant 0, \quad x \in \Gamma,
$$

implies that (4) and (5) hold for all $\phi \in C_{0}^{\infty}\left(\mathbf{R}^{n}\right)$, provided $\Delta_{n} g>0$ on $\Omega$.

Example 2 follows from the fact that $\gamma_{1} g=\nabla g \cdot \nu=r^{-1} \alpha^{\prime}(r)\left(x_{1}, \ldots, x_{n}\right) \cdot \nu$ for any nonzero $x \in \Gamma$.

In some of our applications, when $g$ is a radial function as above, $\alpha^{\prime}(r)$ will be nonnegative. In order to insure that inequality (5) holds for all $\phi \in C_{0}^{\infty}\left(\mathbf{R}^{n}\right)$ we will need to have $\Gamma=\Gamma^{-} \cup \Gamma^{0}$ where $\Gamma^{-}$and $\Gamma^{0}$ are defined as follows:

Define $\Gamma^{+}, \Gamma^{0}$, and $\Gamma^{-}$to be the set of all $x \in \Gamma$ such that

$$
\left(x_{1}, \ldots, x_{n}\right) \cdot\left(\nu_{1}, \ldots, \nu_{n}\right)
$$

is positive, zero, or negative, respectively, where $\nu=\left(\nu_{1}, \ldots, \nu_{n}\right)$ is the unit outward normal vector at $x \in \Gamma$.

Note that $x$ is in $\Gamma^{+}, \Gamma^{0}$, or $\Gamma^{-}$according to whether the angle $\theta$ between the point vector from the origin to $x$ and the outward normal vector $\nu$ is acute, right, or obtuse. For example, if $\Omega$ is the exterior of a ball that is centered at the origin, then $\theta=\pi$ and $\Gamma=\Gamma^{-}$.

Corollary to Lemma 2. If $\phi \in C_{0}^{1}(\Omega \sim\{0\})$, then

$$
4 \int_{\Omega}|x|^{\beta}|\nabla \phi(x)|^{2} d x \geqslant(\beta-2+n)^{2} \int_{\Omega}|x|^{\beta-2}|\phi(x)|^{2} d x .
$$

Moreover, for $n \geqslant 2$, inequality (7) is valid for all $\phi \in\left\{u \in C_{0}^{1}\left(\mathbf{R}^{n} \sim\{0\}\right): u(x)=0\right.$ on $\left.\Gamma^{+}\right\}$when $\beta>2-n$ and when $\beta<2-n$, it is valid for all $\phi \in\{u \in$ $C_{0}^{1}\left(\mathbf{R}^{n} \sim\{0\}\right): u(x)=0$ on $\left.\Gamma^{-}\right\}$.

Proof. The proof is trivial for $\beta=2-n$. If $\beta(\beta-2+n) \neq 0$ let $g(x)=|x|^{\beta}$ and apply Lemma 2. If $\beta=0$, and $n \neq 2$, let $g(x)=(4 /(n-2)) \ln |x|$. The remainder of the proof follows from the discussion above. 
Inequality (7) is proved in [2] for $\phi \in C_{0}^{1}(\Omega \sim\{0\})$ and in that paper an earlier proof is attributed to Piepenbrink [11].

2. Sufficient conditions for discreteness of the spectrum of second order elliptic differential operators. In this section we assume that the boundary of $\Omega, \Gamma$, is a $C^{1}$-manifold and that $\Omega$ is the union of an increasing sequence of bounded open sets $\left\{\Omega_{k}\right\}, \Omega_{k} \subseteq \Omega_{k+1}$, each of which lies on one side of its boundary, $\Gamma_{k}$, which is a $C^{1}$-manifold. We do not assume that $\Omega$ is necessarily bounded.

Define the differential operator $S$ by

$$
S=w(x)^{-1}\left\{-\sum_{i, j=1}^{n} \frac{\partial}{\partial x_{i}} a_{i j}(x) \frac{\partial}{\partial x_{j}}+q(x)\right\}
$$

where $A(x)=\left(a_{i j}(x)\right)$ is a symmetric $n \times n$ matrix whose elements are continuously differentiable on $\Omega$. The weight $w(x)$ is defined as above (see $\mathcal{H} 1$ also), and $q(x)$ is assumed to be a real-valued, measurable, locally integrable function on $\Omega$ that has a positive lower bound on $\Omega$. (The requirement that the lower bound be positive can be relaxed some by adding $\lambda>0$ to the form $\xi$ below.) Let $\sigma(s)$ be a piecewise smooth, nonnegative function on $\Gamma$. Set

$$
\frac{\partial u}{\partial \nu_{A}}=\sum_{i=1}^{n}\left(\sum_{j=1}^{n} a_{i j}(x) \nu_{j}\right) \frac{\partial u}{\partial x_{i}}=(\nabla u)^{t} A \cdot \nu
$$

where $\nu=\left(\nu_{1}, \ldots, \nu_{n}\right)$ denotes the unit outward normal on $\Gamma$. Finally, we define the domain of $S$ to be

$$
\begin{aligned}
& D(S)=\left\{u: u=\phi 1_{\Omega} \text { for some } \phi \in C_{0}^{\infty}\left(\mathbf{R}^{n}\right), S u \in \varrho_{w}^{2}(\Omega), \text { the support of } u\right. \\
&\text { is in } \left.\overline{\Omega_{k}} \text { for some } k, \text { and } c(s) \partial u(s) / \partial \nu_{A}+\sigma(s) u(s)=0 \text { for } s \in \Gamma\right\}
\end{aligned}
$$

where $c(s)$ is equal to either 1 or 0 for each $s \in \Gamma$. Assume that $c(s)$ and $\sigma(s)$ are not both zero at any $s \in \Gamma$.

The differentiable operator $S: \mathscr{L}_{n}^{2}(\Omega) \rightarrow \mathscr{L}_{u}^{2}(\Omega)$ is closable since its domain, which contains $C_{0}^{\infty}(\Omega)$, is dense in $\varrho_{u}^{2}(\Omega)[8$, p. 268]. As a consequence, the form $s$ defined by $s[u, v]=(S u, v)$ with $D(\mathfrak{s})=D(S)$ is closable [8, p. 318] and by Green's formula

$\check{s}[u, v]=\int_{\Omega}[(A(x) \nabla u(x), \nabla v(x))+q(x) u(x) \overline{v(x)}] d x+\int_{\Gamma} \sigma(s) u(s) \bar{v}(s) d s$.

Let $\mathfrak{h}$ be the closure of $\mathfrak{s}$; then $T_{\mathfrak{h}}$ is the Friedrichs extension of $S$.

Let $\mu_{A}$ be a positive-valued function on $[0, \infty)$ satisfying

$$
\text { minimum eigenvalue } A(x) \geqslant \mu_{A}(|x|), \quad x \in \Omega \text {. }
$$

(The eigenvalues of $A(x)$ can be regarded as continuous functions on $\Omega$ - see [8, Chapter II, §5.5].) Suppose that there is a sequence of positive numbers $\left\{c_{k}\right\}$ (possibly converging to zero), such that $\mu_{A}(|x|) \geqslant c_{k}>0, x \in \Omega_{k}$. Note that $\mathfrak{h}$ satisfies $\mathfrak{H} 1$ and $\mathfrak{H} 2$.

Our objective is to use the corollary to Lemmas 1 and 2 to establish conditions on $\mu_{A}(|x|)$ and $w(x)$ that imply that $T_{\mathfrak{b}}$, the Friedrichs extension of $S$, has a discrete 
spectrum. We will do this by exhibiting a function $g(x)$ on $\Omega$ satisfying the hypothesis of Lemma 2, the inequality

$$
\left|\Delta_{n} g\right|^{-1}|\nabla g|^{2} \leqslant \mu_{A}(|x|), \quad x \in \Omega
$$

and the limit $\lim _{k \rightarrow \infty} w(x)\left|\Delta_{n} g\right|^{-1} 1_{\Omega \sim \Omega_{k}}=0$.

Consider the case in which $\Omega$ is unbounded. The differential inequality (8) is simplified considerably if we choose $g(x)$ to be radial, i.e., $g(x)=\alpha(r)$, for $r=|x|$, for some $\alpha \in C^{(2)}(\mathbf{R})$. In this case, $|\nabla g|^{2}=\left|\alpha^{\prime}(r)\right|^{2}$ and $\Delta_{n} g(x)=\alpha^{\prime \prime}(r)+$ $(n-1) r^{-1} \alpha^{\prime}(r)$; hence, inequality (8) reduces to

$$
\left|\alpha^{\prime}(r)\right|^{2} \leqslant \mu_{A}(r)\left(\alpha^{\prime \prime}(r)+(n-1) r^{-1} \alpha^{\prime}(r)\right) .
$$

When $n=1$, it is shown in $[1]($ see $[7 ; 9 ; 4$, p. 120] also for $w(x) \equiv 1)$, where the higher order case is also considered, that for $\Omega=[1, \infty)$ and $q \equiv 0, T_{\mathfrak{h}}$ has a discrete spectrum if and only if

$$
\lim _{x \rightarrow \infty} W(x) \int_{x}^{\infty} \mu_{A}(t)^{-1} d t=0 \quad \text { when } \int_{1}^{\infty} \mu_{A}^{-1} d t<\infty
$$

or

$$
\lim _{x \rightarrow \infty} W(x) \int_{1}^{x} \mu_{A}(t)^{-1} d t=0 \quad \text { when } \int_{1}^{\infty} \mu_{A}^{-1} d t=\infty
$$

where

$$
W(x)= \begin{cases}\int_{x}^{\infty} w(t) d t & \text { when } \int_{1}^{\infty} w(t) d t<\infty \\ \int_{1}^{x} w(t) d t & \text { when } \int_{1}^{\infty} w(t) d t=\infty\end{cases}
$$

This result is extended to the case when $n \geqslant 1$ in the theorems that follow. For the sake of simplicity we assume that $\rho=\inf \{|x|: x \in \Omega\} \geqslant \beta>0$ for some number $\beta$.

TheOREM 1. Let $\Omega$ be unbounded. Assume that either $\Gamma^{+}=\varnothing$ or that $u(s)=0$ on $\Gamma^{+}$for all $u \in D(S)$. Let $\omega(r)$ be a positive-valued, piecewise continuous function on $(0, \infty)$ satisfying

$$
w(x) \leqslant \omega(r), \quad x \in \Omega, r=|x| .
$$

Assume that there is a number $K>0$ such that for all $x \in \Omega$

$$
\int_{r}^{\infty} s^{1-n} \mu_{A}(s)^{-1} d s \int_{\rho}^{r} s^{n-1} \omega(s)\left[\int_{\beta}^{s} t^{n-1} \omega(t) d t \int_{s}^{\infty} t^{1-n} \mu_{A}(t)^{-1} d t\right]^{-1} d s \leqslant K .
$$

If

$$
\lim _{r \rightarrow \infty} \int_{\beta}^{r} t^{n-1} \omega(t) d t \int_{r}^{\infty} t^{1-n} \mu_{A}(t)^{-1} d t=0
$$

then $T_{\mathfrak{h}}$ has a discrete spectrum.

Proof. Let $g(x)=\int_{\rho}^{|x|} r^{1-n}\left[\int_{r}^{\infty} s^{1-n} \mu_{A}(s)^{-1} d s\right]^{-1} d r, x \in \Omega$, and $h(x)=\int_{\rho}^{|x|} r^{1-n} \int_{\rho}^{r} s^{n-1} \omega(s)\left[\int_{\beta}^{s} t^{n-1} \omega(t) d t \int_{s}^{\infty} t^{1-n} \mu_{A}(t)^{-1} d t\right]^{-1} d s d r, \quad x \in \Omega$. 
Then

$$
\begin{gathered}
|\nabla g|^{2}=|x|^{2-2 n}\left[\int_{|x|}^{\infty} s^{1-n} \mu_{A}(s)^{-1} d s\right]^{-2}, \\
\Delta_{n} g=\mu_{A}(|x|)^{-1}|\nabla g|^{2} \\
|\nabla h|^{2}=|x|^{2-2 n}\left[\int_{\rho}^{|x|} s^{n-1} \omega(s)\left(\int_{\beta}^{s} t^{n-1} \omega(t) d t \int_{s}^{\infty} t^{1-n} \mu_{A}(t)^{-1} d t\right)^{-1} d s\right]^{2},
\end{gathered}
$$

and

$$
\Delta_{n} h=\omega(|x|) / \int_{\beta}^{|x|} t^{n-1} \omega(t) d t \int_{|x|}^{\infty} t^{1-n} \mu_{A}(t)^{-1} d t
$$

Hence, for any $u \in D(\mathfrak{s})$

$$
\begin{aligned}
\mathfrak{s}[u, u] & \geqslant \int_{\Omega} \mu_{A}(|x|)|\nabla u|^{2} d x \geqslant \int_{\Omega}|\nabla g|^{2}\left(\Delta_{n} g\right)^{-1}|\nabla u|^{2} d x \\
& \geqslant 2^{-1} \int_{\Omega}|\nabla g||\nabla u||u| d x \geqslant(2 K)^{-1} \int_{\Omega}|\nabla h||\nabla u||u| d x \\
& \geqslant(4 K)^{-1} \int_{\Omega} \Delta_{n} h|u|^{2} d x
\end{aligned}
$$

by Lemma 2 and inequality (11). The conclusion now follows from (10), (12), and the corollary to Lemma 1.

The rather annoying requirement posed by (11) is not very restrictive when viewed in conjunction with (12). (The author conjectures that the requirement (11) can be eliminated.) For example, if the product of (12) decreases to zero then it is easy to show that (11) is satisfied. More generally, it is not hard to show that (11) is satisfied provided there is a function $F(r)$ such that for any $x \in \Omega$

$$
\begin{aligned}
& {\left[\int_{\beta}^{s} t^{n-1} \omega(t) d t \int_{s}^{\infty} t^{1-n} \mu_{A}(t)^{-1} d t\right]^{-1}} \\
& \quad \leqslant F(|x|)+\left[\int_{\beta}^{|x|} t^{n-1} \omega(t) d t \int_{|x|}^{\infty} t^{1-n} \mu_{A}(t)^{-1} d t\right]^{-1}, \quad s \leqslant|x|,
\end{aligned}
$$

and

$$
F(|x|) \int_{\rho}^{|x|} s^{n-1} \omega(s) d s \int_{|x|}^{\infty} s^{1-n} \mu_{A}(s)^{-1} d s
$$

is bounded for all $x \in \Omega$. If $F(r)$ is a constant function then this requirement specifies that as the inverse of the product in (12), $\left[\int_{\beta}^{r} t^{n-1} \omega(t) d t \int_{r}^{\infty} t^{1-n} \mu_{A}(t)^{-1} d t\right]^{-1}$, diverges to infinity, the negative oscillation remains bounded.

The next theorem shows that for certain weight functions an analogous sufficient condition for a discrete spectrum holds even when

$$
\int_{\rho}^{\infty} t^{1-n} \mu_{A}(t)^{-1} d t=\infty
$$


Theorem 2. Let $\Omega$ be unbounded. Assume that either $\Gamma^{-}=\varnothing$ or that $u(s)=0$ on $\Gamma^{-}$for all $u \in D(\mathfrak{I})$. Let inequality (10) hold and $\int_{\sigma}^{\infty} s^{n-1} \omega(s) d s<\infty$. If $n=2$ assume that

$$
\int_{\rho}^{\infty}\left(r \int_{\sigma}^{r} s^{-1} \mu_{A}(s)^{-1} d s\right)^{-1} d r<\infty
$$

If

(13) $\int_{\rho}^{|x|} s^{1-n} \mu_{A}(s)^{-1} d s \int_{|x|}^{\infty} s^{n-1} \omega(s)\left(\int_{s}^{\infty} t^{n-1} \omega(t) d t \int_{\sigma}^{s} t^{1-n} \mu(t)^{-1} d t\right)^{-1} d s$

is bounded on $G$ and

$$
\lim _{r \rightarrow \infty} \int_{r}^{\infty} t^{n-1} \omega(t) d t \int_{\sigma}^{r} t^{1-n} \mu_{A}(t)^{-1} d t=0
$$

Then $T_{\mathfrak{h}}$ has a discrete spectrum.

Proof. Let

$$
\begin{gathered}
g(x)=\int_{|x|}^{\infty} r^{1-n}\left[\int_{\sigma}^{r} s^{1-n} \mu_{A}(s)^{-1} d s\right]^{-1} d r \\
h(x)=-\int_{\rho}^{|x|} s^{1-n} \int_{s}^{\infty} t^{n-1} \omega(t)\left(\int_{t}^{\infty} u^{n-1} \omega(u) d u \int_{\sigma}^{t} u^{1-n} \mu_{A}(u)^{-1} d u\right)^{-1} d t d s
\end{gathered}
$$

and then proceed as in the proof of Theorem 1 .

It should be noted that when $\mu_{A}(t)=t^{2-n}$ and $\omega(t)=t^{\beta}$ for $\beta<-n$ the limit in (14) holds but the function in (13) is not bounded on $\Omega$. However, it is shown below that $T_{\mathfrak{h}}$ has a discrete spectrum even in this case.

Theorems 1 and 2 obviously remain valid if (12) and (14) are replaced by the more general conditions

$$
\lim _{|x| \rightarrow \infty}\left\{w(x)^{-1} q(x)+\left[\int_{\beta}^{|x|} t^{n-1} \omega(t) d t \int_{|x|}^{\infty} t^{1-n} \mu_{A}(t)^{-1} d t\right]^{-1}\right\}=\infty
$$

and

(16) $\lim _{|x| \rightarrow \infty}\left\{w(x)^{-1} q(x)+\left[\int_{|x|}^{\infty} t^{n-1} \omega(t) d t \int_{\sigma}^{|x|} t^{1-n} \mu_{A}(t)^{-1} d t\right]^{-1}\right\}=\infty$,

respectively.

In the special case of $\Omega=\mathbf{R}^{n}$ and $w(x) \equiv 1$, a theorem of Schechter [13, p. 192] (cf. Lemma 3.3 of [14]) can be used to remove condition (11) of Theorem 1. This is achieved by adapting the proof of Theorem 1 as follows:

Let $h(x)=|x|^{2} / 2$ and let $g(x)$ be defined as in the proof of Theorem 1. Suppose that for a given $\varepsilon>0$

$$
|x|^{n} \int_{|x|}^{\infty} r^{1-n} \mu_{A}(r)^{-1} d r<\varepsilon
$$


when $|x|>B$. Then for $\phi \in\left\{u \in C_{0}^{\infty}\left(\mathbf{R}^{n}\right): u(x)=0\right.$ for $\left.|x| \leqslant B\right\}$

$$
\begin{aligned}
\mathfrak{s}(\phi, \phi) & \geqslant \int_{\mathbf{R}^{n}} \mu_{A}(|x|)|\nabla \phi|^{2} d x \geqslant 2^{-1} \int_{\mathbf{R}^{n}}|\nabla g||\nabla \phi||\phi| d x \\
& \geqslant(2 \varepsilon)^{-1} \int_{\mathbf{R}^{n}}|\nabla h||\nabla \phi||\phi| d x \geqslant n(4 \varepsilon)^{-1} \int_{\mathbf{R}^{n}}|\phi|^{2} d x .
\end{aligned}
$$

According to Schechter's theorem, this implies that the essential spectrum of $T_{\mathfrak{h}}$ is in $\left[n(4 \varepsilon)^{-1}, \infty\right)$. The result follows.

Consequently, we can conclude from the corollary to Theorem 4 below that when $\Omega=\mathbf{R}^{n}, q(x) \equiv 0, w(x) \equiv 1$, and min e.v. $A(x)=\max$ e.v. $A(x)=\mu_{A}(|x|)$, then

$$
\lim _{|x| \rightarrow \infty}|x|^{n} \int_{|x|}^{\infty} r^{1-n} \mu_{A}(r)^{-1} d r=0
$$

is necessary and sufficient in order that the spectrum of $T_{\mathrm{h}}$ be discrete.

Finally, note that in the case of $\Omega$ bounded, results like Theorems 1 and 2 can be established as well via Lemmas 1 and 2. For example, if $\Gamma$ included a portion of the surface of the ball $|x|<\lambda$ and the singularities of the coefficients occur on that part of $\Gamma$, then with a suitable choice of $\left\{\Omega_{k}\right\}$, Theorems 1 and 2 would hold with $\infty$ replaced by $\lambda$.

3. Necessary conditions for discreteness of the spectrum of second order elliptic differential operators. In this section, we show that Theorems 1 and 2 are sharp, at least in certain cases. Our main device for doing this will be the following theorem that can be found in the book of Glazman [4, p. 15].

THEOREM 3. A necessary and sufficient condition for the number of points of the spectrum of a selfadjoint operator $A$, lying to the left of a given point $\lambda_{0}$, to be an infinite set, is that there exists an infinite dimensional set $G \subset D(A)$ for which

$$
\left(A u-\lambda_{0} u, u\right)<0 \quad \text { for all } u \in G .
$$

Since $\mathfrak{s}$ of $\S 2$ is symmetric then $T_{\mathfrak{h}}$ is selfadjoint $[8$, p. 323] and Theorem 3 applies to $T_{\mathrm{l}}$.

Corollary to Theorem 3. If there is an infinite dimensional set $M \subset D(\mathfrak{h})$ such that

$$
\mathfrak{h}(u, u)<\lambda_{0}(u, u) \quad \text { for all } u \in M
$$

then the number of points of the spectrum of $T_{\mathrm{h}}$, lying to the left of $\lambda_{0}$, is infinite.

Proof. Let $\left\{\phi_{i}\right\}_{i=1}^{\infty}$ be an orthonormal basis for $M$ and let $G=D\left(T_{\mathfrak{h}}\right) \cap M$. Since $\left(T_{\mathfrak{h}} v, v\right)=\mathfrak{h}(v, v)$ for all $v \in D\left(T_{\mathfrak{h}}\right)$ then we need only show that $G$ is infinite dimensional.

Suppose $G$ is finite dimensional; then for some $k,\left(\phi_{k}, v\right)=0$ for all $v \in G$. According to the first representation theorem [8, p. 322] (by Friedrichs for this special case) $D\left(T_{\mathfrak{h}}\right)$ is a core of $\mathfrak{h}$. Therefore, there is a sequence $\left\{\psi_{j}\right\}_{j=1}^{\infty} \subseteq D\left(T_{\mathfrak{h}}\right)$ converging to $\phi_{k}$ in $\mathcal{L}_{w}^{2}(\Omega)$ and

$$
\mathfrak{h}\left(\psi_{j}, \psi_{j}\right) \rightarrow \mathfrak{h}\left(\phi_{k}, \phi_{k}\right) \quad \text { as } j \rightarrow \infty
$$


Consequently, for $j$ large $\mathfrak{h}\left(\psi_{j}, \psi_{j}\right)<\lambda_{0}\left(\psi_{j}, \psi_{j}\right)$ which implies that $\psi_{j} \in G$. But $\psi_{j} \rightarrow \phi_{k}$ in $\mathcal{L}_{w}^{2}(\Omega)$ implies that

$$
\left(\phi_{k}, \psi_{j}\right) \rightarrow\left(\phi_{k}, \phi_{k}\right)=1 \text { as } j \rightarrow \infty,
$$

which contradicts the fact that $\left(\phi_{k}, v\right)=0$ for all $v \in G$. The proof is complete.

Lemma 3. Let $U$ be a bounded open subset of $\Omega$ that lies on one side of its boundary, which is a $C^{1}$ manifold. Suppose $\phi \in C_{0}(U)$ has a piecewise continuous derivative in $U$. If $q$ and $w$ are bounded on compact subsets of $\Omega$, then $\phi \in D(\mathfrak{h})$.

Proof. Let $\tilde{\phi}$ be the extension of $\phi$ as zero outside $U$. Since $\partial \tilde{\phi} / \partial x_{i}$ has at most jump discontinuities, then $\tilde{\phi}, \partial \tilde{\phi} / \partial x_{i} \in \mathfrak{L}^{2}\left(\mathbf{R}^{n}\right)$, for $i=1,2, \ldots, n$. Hence, $\tilde{\phi} \in H^{1}\left(\mathbf{R}^{n}\right)$ which implies that $\phi=\tilde{\phi} 1_{U} \in H_{0}^{1}(U)$ (see Treves [16, pp. 245-247]). Consequently, $\phi$ is the $H^{1}(U)$-limit of $C_{0}^{\infty}(U)$ functions $\left\{\psi_{k}\right\}_{k=1}^{\infty}$. Since $q$ and $w$ are bounded on supp $\phi$ and each $a_{i j} \in C^{1}(\Omega)$, then $\left\{\psi_{k}\right\}$ is $\mathfrak{h}$-convergent to $\phi[8, \mathrm{p}$. 313] which implies that $\phi \in D(\mathfrak{h})$ by Theorem 1.17 of $[8$, p. 315].

In order to prove necessary conditions for the discreteness of the spectrum of $T_{\mathfrak{h}}$ analogous to the sufficient conditions of $\S 1$, the following hypothesis will be required concerning $\Omega$.

$\mathcal{H}$ 3. Let $\left\{x \in \mathbf{R}^{n}:|x| \geqslant \beta\right\} \subseteq \Omega$ for some number $\beta>1$.

TheOREM 4. Assume $\mathcal{H} 3$. Let $\gamma_{A}(r), \tilde{\omega}(r), \tilde{q}(r) \in C[\beta, \infty)$ and assume that for $x \in\left\{x \in \mathbf{R}^{n}:|x| \geqslant \beta\right\}$

$$
\text { maximum eigenvalue } A(x) \leqslant \gamma_{A}(|x|), \quad w(x) \geqslant \tilde{\omega}(|x|),
$$

and

$$
q(x) \leqslant \tilde{q}(|x|) \text {. }
$$

If there is a sequence $\left\{\phi_{k}(r)\right\}$ of continuous piecewise differentiable functions with disjoint supports in $[\beta, \infty)$ such that

$$
\int_{\beta}^{\infty} r^{n-1}\left[\gamma_{A}(r)\left|\phi_{k}^{\prime}(r)\right|^{2}+\left(\tilde{q}(r)-\lambda_{0} \tilde{\omega}(r)\right)\left|\phi_{k}(r)\right|^{2}\right] d r<0
$$

for each $k$, then spectrum $\left(T_{\mathfrak{h}}\right) \cap\left(-\infty, \lambda_{0}\right)$ is infinite.

Proof. The proof follows from the corollary to Theorem 3, Lemma 3, the inequality

$$
\begin{aligned}
h\left[\phi_{k}, \phi_{k}\right] & -\lambda_{0}\left(\phi_{k}, \phi_{k}\right) \\
& \leqslant \int_{\Omega}\left[\gamma_{A}(|x|)\left|\phi_{k}^{\prime}(|x|)\right|^{2}+\left(\tilde{q}(|x|)-\lambda_{0} \tilde{\omega}(|x|)\right)\left|\phi_{k}(|x|)\right|^{2}\right] d x
\end{aligned}
$$

for each $k$, and a change of variable to polar coordinates.

It is interesting to note that for $\gamma_{A}^{\prime}(r) \in C[\beta, \infty)$, the sequence $\left\{\phi_{k}\right\}$ of Theorem 4 exists such that (17) is satisfied if and only if the ordinary differential equation

$$
-\frac{d}{d r}\left(r^{n-1} \gamma_{A}(r) \frac{d}{d r} y(r)\right)+r^{n-1} \tilde{q}(r) y(r)=r^{n-1} \lambda_{0} \tilde{\omega}(r) y(r)
$$

is oscillatory [4] (i.e., every solution has an infinite number of zeros on $[\beta, \infty)$ ) or, equivalently, if each selfadjoint differential operator generated by (18) has an 
infinite number of points of its spectrum in the interval $\left(-\infty, \lambda_{0}\right)$. (The continuity requirements on the coefficients probably can be relaxed.) It is known (cf. Ahlbrandt, Hinton and Lewis [1], Moore [10] and Hille [5]) that equation (18) is oscillatory provided $\tilde{q}(r) \leqslant \lambda_{0} \tilde{\omega}(r)$ on $[\beta, \infty)$ and either

$$
\int_{\beta}^{\infty} r^{n-1}\left(\lambda_{0} \tilde{\omega}(r)-\tilde{q}(r)\right) d r<\infty
$$

and

$$
\lim _{r \rightarrow \infty} \sup \int_{\beta}^{r} s^{1-n} \gamma_{A}(s)^{-1} d s \int_{r}^{\infty} s^{n-1}\left(\lambda_{0} \tilde{\omega}(s)-\tilde{q}(s)\right) d s>1
$$

or

$$
\int_{\beta}^{\infty} s^{1-n} \gamma_{A}(s)^{-1} d s<\infty
$$

and

$$
\lim _{r \rightarrow \infty} \sup \int_{r}^{\infty} s^{1-n} \gamma_{A}(s)^{-1} d s \int_{\beta}^{r} s^{n-1}\left(\lambda_{0} \tilde{\omega}(s)-\tilde{q}(s)\right) d s>1 .
$$

Also, equation (18) is oscillatory [10] when

$$
\int_{\beta}^{\infty} s^{1-n} \gamma_{A}(s)^{-1} d s=\int_{\beta}^{\infty} s^{n-1}\left(\lambda_{0} \tilde{\omega}(s)-\tilde{q}(s)\right) d s=\infty .
$$

Consequently, corresponding to the sufficient condition for discreteness of the spectrum of $T_{\mathrm{b}}$ given in Theorem 1 we have the necessary conditions of the next corollary.

Corollary to Theorem 4. Assume $\mathfrak{H} 3, \tilde{q}(r) \equiv 0$ on $\Omega$, and that $\gamma_{A}^{\prime}(r) \in C[\beta, \infty)$. Suppose that $T_{\mathrm{b}}$ has a discrete spectrum. If $\int_{\beta}^{\infty} r^{n-1} \tilde{\omega}(r) d r<\infty$, then

$$
\lim _{r \rightarrow \infty} \int_{\beta}^{r} s^{1-n} \gamma_{A}(s)^{-1} d s \int_{r}^{\infty} s^{n-1} \tilde{\omega}(s) d s=0 .
$$

If $\int_{\beta}^{\infty} s^{1-n} \gamma_{A}(s)^{-1} d s<\infty$, then

$$
\lim _{r \rightarrow \infty} \int_{\beta}^{r} s^{n-1} \tilde{\omega}(s) d s \int_{r}^{\infty} s^{1-n} \gamma_{A}(s)^{-1} d s=0
$$

Proof. Since the spectrum of $T_{\mathrm{h}}$ is discrete then neither (19) nor (20) holds for any $\lambda_{0}>0$, i.e., either

$$
\lim _{r \rightarrow \infty} \sup \int_{\beta}^{r} s^{1-n} \gamma_{A}(s)^{-1} d s \int_{r}^{\infty} s^{n-1} \tilde{\omega}(s) d s \leqslant \lambda_{0}^{-1}
$$

for every $\lambda_{0}>0$ or

$$
\lim _{r \rightarrow \infty} \sup \int_{r}^{\infty} s^{1-n} \gamma_{A}(s)^{-1} d s \int_{\beta}^{r} s^{n-1} \tilde{\omega}(s) d s \leqslant \lambda_{0}^{-1}
$$

for every $\lambda_{0}>0$, depending on which integral exists. Consequently, the conclusion follows.

In order to investigate the sharpness of Theorems 1 and 2 and the corollary to Theorem 4, we consider the following example. 
EXAMPLE 3. Let $\mu_{A}(r)=r^{\alpha_{1}}, \gamma_{A}(r)=r^{\alpha_{2}}, \omega(r)=\tilde{\omega}(r)=r^{\kappa}$, and $q(x)=|x|^{\tau}=r^{\tau}$ for $r=|x|$.

(i) Sufficient conditions. If $\alpha_{1}>2-n$ assume that $u=0$ on $\Gamma^{+}$, if $\alpha_{1}=2-n$ assume that $u=0$ on $\Gamma$, and if $\alpha_{1}<2-n$ assume that $u=0$ on $\Gamma^{-}$for all $u \in D(S)$. The spectrum of $T_{\mathrm{h}}$ is discrete if $\alpha_{1}>\kappa+2$ or $\tau>\kappa$.

(ii) Necessary conditions. Assume $\mathcal{H} 3$. If the spectrum of $T_{\mathfrak{h}}$ is discrete then $\alpha_{2}>\kappa+2$ or $\tau>\kappa$.

Proof. Theorems 1 and 2 with (15) and (16) replacing (12) and (14) imply (i) when $\alpha_{1} \neq 2-n$. If $2-n=\alpha_{1}>\kappa+2$, then $\kappa=-n-2 \delta$ for some $\delta>0$. By the corollary to Lemma 2

$$
\int_{\Omega}|x|^{2-n}|\nabla \phi|^{2} d x \geqslant \int_{\Omega}|x|^{2-n-\delta}|\nabla \phi|^{2} d x \geqslant \frac{\delta^{2}}{4} \int_{\Omega}|x|^{-n-\delta}|\phi|^{2} d x .
$$

The discreteness of the spectrum of $T_{\mathfrak{h}}$ follows from the corollary to Lemma 1 .

In order to prove (ii) we need to show that the equation

$$
-\left(r^{\alpha_{2}+n-1} y^{\prime}(r)\right)^{\prime}+r^{n-1}\left(r^{\tau}-\lambda_{0} r^{\kappa}\right) y(r)=0
$$

is oscillatory for some $\lambda_{0}>0$ when $\alpha_{2} \leqslant \kappa+2$ and $\tau \leqslant \kappa$. If $\kappa \neq-n$, this fact follows from the oscillation criteria (19) and (20). If $\kappa=-n$, it follows from (21).

Therefore, in the case of the Dirichlet problem $-c(s) \equiv 0$ and $\sigma(s) \equiv 1$ on $\Gamma$ - and

$$
S=-|x|^{-\kappa}\left\{\sum_{i=1}^{n} \frac{\partial}{\partial x_{i}}|x|^{\alpha} \frac{\partial}{\partial x_{i}}+|x|^{\tau}\right\}
$$

with hypothesis $\mathcal{H} 3$ holding; the spectrum of $T_{\mathfrak{h}}$ is discrete if and only if $\alpha>\kappa+2$ or $\tau>\kappa$.

ACKNOWLEDGEMENT. The author wishes to express his gratitude for many fruitful discussions he has had with Robert M. Kauffman and Ian W. Knowles concerning this paper.

ADDED IN PROOF. Kalf and Walter in their paper, Strongly singular potentials and essential self-adjointness of singular elliptic operators in $C_{0}^{\infty}\left(\mathbf{R}^{n} \backslash\{0\}\right)$ (J. Funct. Anal. 10 (1972)), prove an inequality which is a special case of Lemma 2 -choose $g(x)$ as in the proofs of Theorems 1 and 2.

\section{REFERENCES}

1. Calvin D. Ahlbrandt, Don B. Hinton and Roger T. Lewis, Necessary and sufficient conditions for the discreteness of the spectrum of certain singular differential operators, Canad. J. Math. 33 (1981), 229-246.

2. W. Allegretto, Nonoscillation theory of elliptic equations of order $2 n$, Pacific J. Math. 64 (1976), 1-16.

3. N. Dunford and J. T. Schwartz, Linear operators. II, Interscience, New York, 1957.

4. I. M. Glazman, Direct methods of qualitative spectral analysis of singular differential operators, Israel Program for Scientific Translations, Jerusalem, 1965.

5. Einar Hille, Nonoscillation theorems, Trans. Amer. Math. Soc. 64 (1948), 234-252.

6. Don B. Hinton and Roger T. Lewis, Discrete spectra criteria for singular differential operators with middle terms, Math. Proc. Cambridge Philos. Soc. 77 (1975), 337-347.

7. G. A. Kalyabin, $A$ necessary and sufficient condition for the spectrum of a homogeneous operation to be discrete in the matrix case, Differential Equations 9 (1973), 951-954.

8. T. Kato, Perturbation theory for linear operators, 2nd ed., Springer-Verlag, Berlin, Heidelberg and New York, 1976. 
9. Roger T. Lewis, The discreteness of the spectrum of self-adjoint, even order, one-term, differential operators, Proc. Amer. Math. Soc. 42 (1974), 480-482.

10. Richard A. Moore, The behavior of solutions of a linear differential equation of second order, Pacific J. Math. 5 (1955), 125-145.

11. J. Piepenbrink, Integral inequalities and theorems of Liouville type, J. Math. Anal. Appl. 26 (1969), 630-639. MR 39 \#3136.

12. Franz Rellich, Perburbation theory of eigenvalue problems, Gordon \& Breach, New York, 1969.

13. Martin Schechter, Spectra of partial differential operators, North-Holland, Amsterdam, 1971.

14. , On the spectra of singular elliptic operators, Mathematika 23 (1976), 107-115.

15. R. E. Showalter, Hilhert space methods for partial differential equations, Pitman, New York, 1977.

16. François Treves, Basic linear partial differential equations, Academic Press, New York, 1975.

Department of Mathematics, University of Alabama in Birmingham, Birmingham, Alabama 35294 